2. National Ski Areas Association. Helmet usage and safety fact sheet. Available at: https://www.nsaa.org/NSAA/ resources/Industry_Stats. Accessed November 11, 2020.

3. Russell K, Christie J, Hagel BE. The effect of helmets on the risk of head and neck injuries among skiers and snowboarders: a meta-analysis. CMAJ. 2010;182(4): $333-40$.

4. Haider AH, Saleem T, Bilaniuk JW, Barraco RD. An evidence-based review: efficacy of safety helmets in reduction of head injuries in recreational skiers and snowboarders. J Trauma Acute Care Surg. 2012;73(5): 1340-7.

5. Bailly N, Laporte JD, Afquir S, Masson C, Donnadieu T, Delay JB, et al. Effect of helmet use on traumatic brain injuries and other head injuries in alpine sport. Wilderness Environ Med. 2018;29(2):151-8.

6. Sulheim S, Ekeland A, Holme I, Bahr R. Helmet use and risk of head injuries in alpine skiers and snowboarders: changes after an interval of one decade. Br J Sports Med. 2017;51(1):44-50.

7. Ruedl G, Kopp M, Burtscher M, Zorowka P, Weichbold V, Stephan K, et al. Effect of wearing a ski helmet on perception and localization of sounds. Int J Sports Med. 2014;35(8):645-50.

8. Cullen AP. Ozone depletion and solar ultraviolet radiation: ocular effects, a United Nations environment programme perspective. Eye Contact Lens. 2011;37(4):185-90.

9. Ivanov IV, Mappes T, Schaupp P, Lappe C, Wahl S. Ultraviolet radiation oxidative stress affects eye health. J Biophotonics. 2018;11(7):e201700377.

10. MacEwen CJ. Sport associated eye injury: a casualty department survey. Br J Ophthalmol. 1987;71(9):701-5.

\section{Toxic Brain Edema and Brain Death After Scorpion Envenomation}

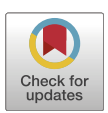

\section{To the Editor:}

Scorpion sting is common in some global regions and mostly causes local envenoming of varying severity. Some estimates suggest that perhaps only $10 \%$ of those stung by even the most medically important species develop severe systemic envenomation; however, the frequency of severe envenomation is higher in children. With few exceptions, systemic envenomation by medically important species is characterized by relatively similar neurotoxic stimulation syndromes. ${ }^{1}$

Here, we report a 12-y-old girl who developed toxic encephalopathy and lethal brain edema owing to scorpion envenomation (Leiurus abdullahbayrami [Scorpiones: Buthidae]). She was transferred to our pediatric intensive care unit from another center after 2 $\mathrm{d}$ of follow-up. Based on the documents provided by the other medical unit, the patient was intubated 1 to $2 \mathrm{~h}$ after the scorpion sting owing to confusion and respiratory distress and was given tetanus vaccine, 1 vial of antivenom (polyvalent antiscorpion antivenom, Refik
Saydam Hygiene Center, Ankara, Turkey), and doxazosin therapy in the initial treating facility. Because the patient experienced cardiac arrest for $5 \mathrm{~min}$, cardiopulmonary resuscitation was performed. When the ejection fraction of the left ventricle was $30 \%$ on echocardiography, she was referred to us with a diagnosis of toxic myocarditis.

Immediately after admission to our unit, she was intubated and had decompensated shock findings. Her Glasgow Coma Scale score was 6 of 15. Blood gas findings showed mild acidosis. Laboratory results showed white blood cell levels were $15.73 \times 10^{3} \cdot \mu \mathrm{L}^{-1}$, hemoglobin was $11.9 \mathrm{~g} \cdot \mathrm{dL}^{-1}$, platelets was $254 \times 10^{3} \cdot \mu \mathrm{L}^{-1}$, serum creatinine was $0.87 \mathrm{mg} \cdot \mathrm{dL}^{-1}$, blood urea nitrogen was 24 $\mathrm{mg} \cdot \mathrm{dL}^{-1}$, aspartate aminotransferase was $244 \mathrm{U} \cdot \mathrm{L}^{-1}$, alanine aminotransferase was $71 \mathrm{U} \cdot \mathrm{L}^{-1}$, creatinine kinase was $8191 \mathrm{U} \cdot \mathrm{L}^{-1}$, troponin $\mathrm{T}$ was $1924 \mathrm{pg} \cdot \mathrm{mL}^{-1}$ (normal range: $0-14)$, $\mathrm{N}$-terminal pro-brain natriuretic peptide was $>35 \mathrm{ng} \cdot \mathrm{L}^{-1}$, and $\mathrm{CK}-\mathrm{MB}$ was $75 \mathrm{ng} \cdot \mathrm{mL}^{-1}$ (normal range: 0-3.6); international normalized ratio was 1.59 ; activated thromboplastin time was $24.9 \mathrm{~s}$; prothrombin time (PT) was $18.4 \mathrm{~s}$; D-dimer was $3789 \mathrm{ng} \cdot \mathrm{mL}^{-1}$ (normal range: $0-243$ ); and fibrinogen was $5.58 \mathrm{~g} \cdot \mathrm{L}^{-1}$ (normal range: 2-3.93).

To stabilize the patient, we continued with maintenance fluid, milrinone, and doxazosin therapies and dobutamin to correct hypotension. On the first day of admission, the patient was given 1 more vial of Refik Saydam Hygiene Center polyvalent antiscorpion antivenom therapy. On echocardiography, the ejection fraction was 45 to $50 \%$. Cranial computed tomography (CT) was performed on the third day of admission, with decreased alertness and a Glasgow Coma Scale score of 4. The CT report revealed existing widespread hypodense areas in both cerebral hemispheres, loss of gray and white matter separation, and deletion in sulcal structures compatible with edema (owing to possible toxicologic effects) (Figure 1). Because the patient had brain edema, $0.1 \mathrm{~cm}^{3} \cdot \mathrm{kg}^{-1} \cdot \mathrm{h}^{-1} 3 \% \mathrm{NaCl}$ was started. The patient's neurologic condition worsened gradually. On the 10th day of hospitalization, neurologic examination revealed absence of all brainstem activity. CT angiography was performed, and the report was compatible with brain death.

It has been reported that after scorpion sting, cortical disruption may develop before other organ disruptions. ${ }^{2}$ One study with 9 fatal cases documented central nervous system (CNS) symptoms before terminal hypotension and cardiac arrest. ${ }^{3}$ Lethal cerebral edema occurred in a 2-y-old male patient who had diffuse brain edema and ischemic changes on CT after scorpion sting and was later reported to be diagnosed with brain death. ${ }^{4}$ Similarly, our patient had diffuse brain edema 


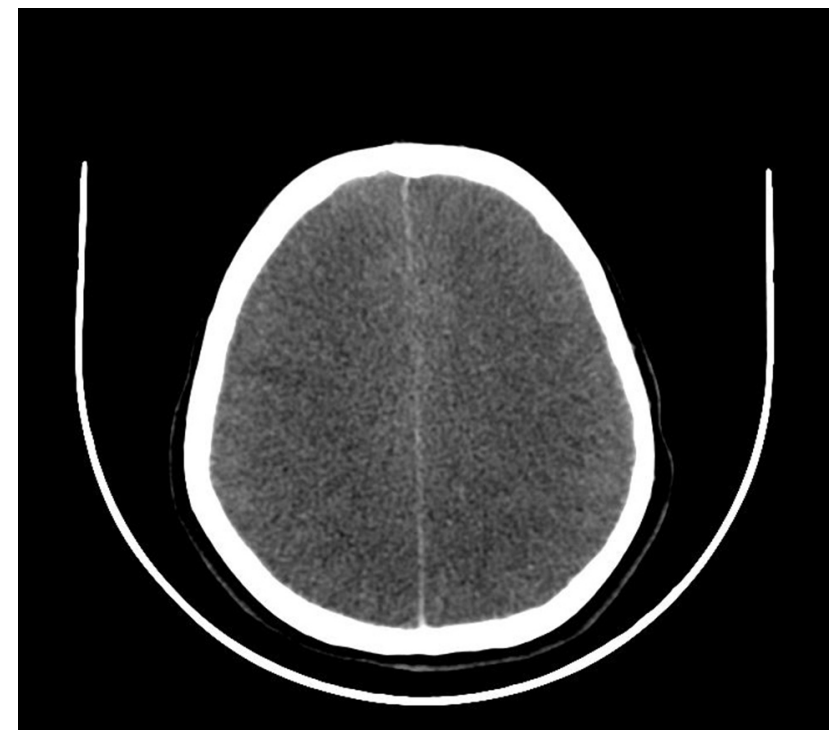

Figure 1. On the third day of admission, cranial computed tomography revealed widespread hypodense areas in both cerebral hemispheres, loss of gray and white matter separation, and deletion in sulcal structures compatible with severe edema.

(owing to possible toxicologic effects) on CT and was diagnosed with brain death on the 10th day of admission. CNS pathophysiologic effects have been hypothetically assigned to venom penetration through the blood-brain barrier because of possible increased permeability in young children. ${ }^{3}$ Symptoms of systemic envenomation may be caused by the venom (toxins) itself or neurotransmitters (catecholamines) and proinflammatory cytokines released owing to the venom. ${ }^{4}$

In conclusion, we believe that this patient had unusually severe brain damage either owing to venomrelated direct CNS toxicity (increased permeability of the blood-brain barrier, affecting the CNS neurons) or the secondary effect of proinflammatory cytokines and neurotransmitters affecting blood vessels, which induced brain ischemia and cytolytic brain edema.

Emrah Gün, MD

Edin Botan, MD

Division of Pediatric Critical Care Medicine Ankara University Faculty of Medicine Ankara, Turkey

Ömer Bektaş, MD Division of Pediatric Neurology Ankara University Faculty of Medicine Ankara, Turkey
Tanıl Kendirli

Division of Pediatric Critical Care Medicine Ankara University Faculty of Medicine

Ankara, Turkey

\section{References}

1. Isbister GK, Bawaskar HS. Scorpion envenomation. N Engl J Med. 2014;371(5):457-63.

2. Ismail M, Abd-Elsalam MA, Morad AM. Do changes in body temperature following envenomation by the scorpion Leiurus quinquestriatus influence the course of toxicity? Toxicon. 1990;28(11):1265-84.

3. Ismail M, Fatani AJ, Dabees TT. Experimental treatment protocols for scorpion envenomation: a review of common therapies and an effect of kallikrein-kinin inhibitors. Toxicon. 1992;30(10):1257-79.

4. Cavari Y, Lazar I, Shelef I, Sofer S. Lethal brain edema, shock, and coagulopathy after scorpion envenomation. Wilderness Environ Med. 2013;24(1):23-7.

\section{Methoxyflurane May Be a Suitable Analgesic for Extreme Prehospital Conditions}

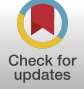

\section{To the Editor:}

Methoxyflurane (MEOF) is a fluorinated hydrocarbon anesthetic that was introduced to the market by Abbott Laboratories as Penthrane in the early 1960s. MEOF was found to have analgesic properties at subanesthetic doses. A disposable inhaler was developed for selfadministration of MEOF for pain relief in minor surgical procedures and obstetrics in the late 1960s. In 1966, the first report of postoperative nephrotoxicity associated with MEOF emerged. Although MEOF has been withdrawn for use as an anesthetic agent, low-dose MEOF delivered via a handheld inhaler has continued to be in wide use in Australia and New Zealand since 1975. MEOF as an analgesic has been used by more than 6 million people over the course of $40 \mathrm{y}$ in Australia and New Zealand. Common nonserious adverse effects are reactions such as headache, nausea, dizziness, and somnolence. Generally, adverse effects are mild and transient, resolving after inhalation is stopped. MEOF administered at analgesic doses via a disposable inhaler has recently become available in Europe for emergency treatment of moderate-to-severe pain in conscious adult trauma patients.

A recent study demonstrates that prehospital pain management is characterized by ongoing oligoanalgesia owing to undelivered analgesia. ${ }^{1}$ Data from the control arms of the studies that compared MEOF to standard 